ERR A T UM

V. Taranenko $\cdot$ R. Meckbach $\cdot$ M. O. Degteva $\cdot$

N. G. Bougrov • Y. Göksu • M. I. Vorobiova · P. Jacob

\title{
Verification of external exposure assessment for the upper Techa riverside by luminescence measurements and Monte Carlo photon transport modeling
}

Published online: 5 December 2003

(C) Springer-Verlag 2003

\section{Radiat Environ Biophys (2003) 42:17-26}

Figure 2: The wrong prefix multiplier for the dose rate was used in Fig. 2; the correct one is $\mu \mathrm{Gy}$ day $^{-1}$

Figure 6: The first sentence in the caption of Fig. 6 has been corrected. It should read: Height dependence of contributions to the cumulative anthropogenic dose in bricks from the river model (on the right) and the reservoir model (on the left).

The online version of the original article can be found at http://dx.doi.org/10.1007/s00411-003-0183-x

V. Taranenko ( $\bullet$ ) R. Meckbach · Y. Göksu · P. Jacob Institute of Radiation Protection,

GSF - National Research Center for Environment and Health, 85764 Neuherberg, Germany

e-mail: taranenko@gsf.de

Tel.: +49-89-31873359

Fax: +49-89-31873363

M. O. Degteva · N. G. Bougrov · M. I. Vorobiova Urals Research Center for Radiation Medicine, 454076 Chelyabinsk, Russian Federation

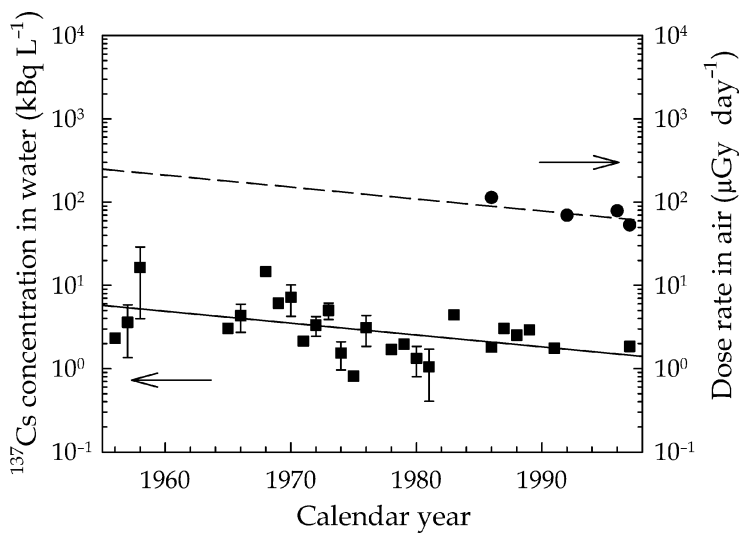

Fig. 2 Measurements of ${ }^{137} \mathrm{Cs}$ concentration in the water of Reservoir 10 (squares) and gamma dose rate measurements near the shore (circles) in Metlino

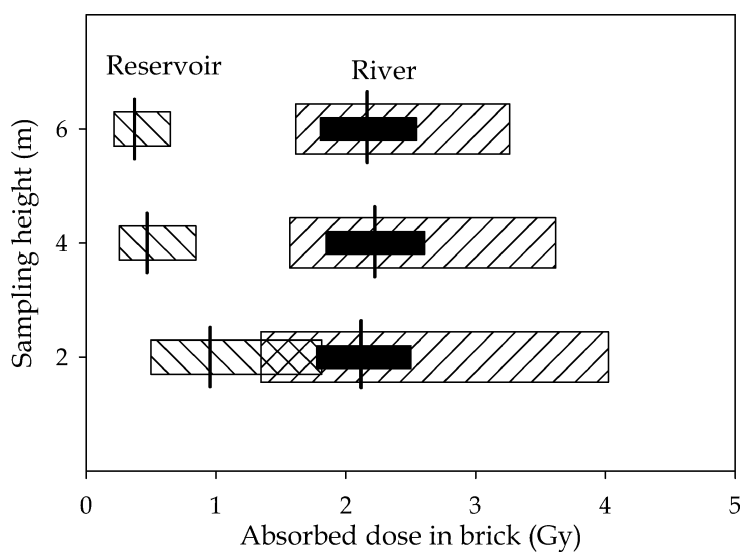

Fig. 6 Height dependence of contributions to the cumulative anthropogenic dose in bricks from the river model (on the right) and the reservoir model (on the left). Vertical lines denote best estimates of deterministic reconstruction. Black boxes represent 95\% CIs considering uncertainty of DRA data for 1949-1956. Shaded boxes represent $95 \%$ CIs for probabilistically reconstructed dose. The assigned heights are relative to the present water level in Reservoir 10 